\title{
GLOBAL HEALTH \& TUBERCULOSIS: A RETROSPECTIVE STUDY
}

\author{
Chattu VK ${ }^{1}$,Alzohairy $\mathrm{M}^{2}$, Sahsah $\mathrm{B}^{3}$, Barsamin $\mathrm{F}^{3}$, Albelaihi $\mathbf{M}^{3}$ \\ ${ }^{1}$ College of Health Sciences, Unversity of Buraimi, Al Buraimi, Oman \\ ${ }^{2}$ College of Applied Medical Sciences, Qassim University, Saudi Arabia \\ ${ }^{3}$ Buraidah Central Hospital, Al Qassim
}

\begin{abstract}
Introduction: A retrospective study to explore the epidemiology and clinical characteristics of Tuberculosis among Saudis, migrant workers from SAARC and other nations. The objective of this study is to study the prevalence and clinical features of Tuberculosis among Saudis \& other migrant workers in central Saudi Arabia.
\end{abstract}

Methodology: A Retrospective study was conducted based on the secondary data pertaining to the patients registered at Regional TB center in Buraidah Central Hospital from Jan 2005 to December 2009.

Results: A total of 355 case records were included of which 187 cases are from Saudi Arabia and remaining 168 were from South Asian countries. Cough with expectoration, fever with evening rise, loss of appetite were the chief clinical presentations. Out of the total non Saudi patients, the Indonesian patients contribute to $74(21 \%)$ followed by Indians $39(11 \%)$, Nepalese $12(3.4 \%)$, Philippines \& Bangladesh with 9 cases each $(2.5 \%)$, and Pakistan with 8 cases $(2.3 \%)$. Among the total cases, there were $341(96 \%)$ new cases, $12(3.4 \%)$ relapse cases and $2(0.6 \%)$ defaulters.

Conclusion: Prevalence of TB among migrant is relatively high. Preventive measures for early diagnosis should be performed especially among migrant workers from countries with high-prevalence of Tuberculosis.

Key words: Epidemiology, Migrant, Prevalence, SAARC nationals, Tuberculosis

\section{INTRODUCTION}

Tuberculosis (TB) has troubled humankind throughout history. Tuberculosis (TB) is an ancient disease that has affected mankind for more than 4,000 years. It is a chronic disease caused by the bacillus Mycobacterium tuberculosis and spreads from person to person through air. TB usually affects the lungs but it can also affect other parts

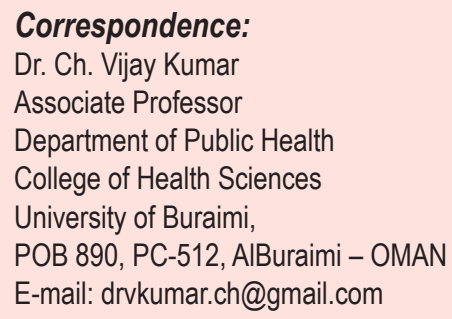

of the body, such as brain, intestines, kidneys, or the spine. Although a declining trend was observed in most developed countries, this was not evident in many developing countries. Global health is now considered important for national and international security, domestic and global economic well-being. In 2011, there were an estimated 8.7 (range, 8.39.0 million) million incident cases (equivalent to 125 cases per 100000 population) of TB globally. ${ }^{1}$ M. tuberculosis is known to be the leading cause of death due to a single infectious agent. ${ }^{2,3 \& 4}$ The worldwide annual incidence continues to increase in Africa with $85 \%$ new cases because of the human immune deficiency virus (HIV) epidemic, whereas it is stable or falling in all other regions. ${ }^{1}$ Although the majority of infected individuals don't exhibit overt signs of disease, they represent a 
large pool of infection that allows for new cases to arise and have a risk of reactivation at a later time in their lives. ${ }^{6}$ The risk increases significantly when the immune system of infected individual becomes suppressed, such as individuals infected with HIV. ${ }^{7,8}$

An accurate description of TB is difficult to obtain because of poor diagnostic facilities and reporting systems in many countries where infection is dominant. ${ }^{9}$ The best information available on global TB comes from the World Health Organization (WHO) with Southeast Asia holding the highest number of people infected with TB in the world.

Cases of pulmonary TB constituted $70.6 \%$ while the extra-pulmonary TB cases constituted $29.4 \%$ of the total cases in 2006.10 In addition, Saudi Arabia in general with its developmental projects is known to attract a lot of international work force from Asian and African countries where infections might be dominant. Therefore, this study was carried out aiming to determine epidemiological and clinical characteristics of TB cases among general population in Qassim region, Saudi Arabia between January 2005 and December 2009 and to provide valuable insight on pulmonary and extrapulmonary TB. Saudi Arabia has a population of 28 million of which 8 million (28.5\%) is contributed by the migrant population mainly from India, Pakistan, Bangladesh, Sri Lanka, Philippines \& Egypt. The remarkable economic and social developments and the large investments in health care services over the past 20 years are expected to have a positive impact on the incidence of TB in Saudi Arabia. Unfortunately, TB control seems to be facing challenges in several regions. The high influx of workers from high burden countries and the large volume of religious visitors together with many illegal immigrants are some of the challenges. There may also be some defects in TB control strategies that need to be evaluated and improved. ${ }^{11}$

\section{METHODOLOGY}

\section{Study population and Data collection:}

This study was conducted in a regional TB center in Qassim region that lies approximately at the centre of the Arabian Peninsula. It was conducted as a retrospective study based on the secondary data pertaining to the patients registered in hospital from January 2005 to December 2009. The study got ethical clearance from the Ethics \& Research Committee of Qassim University and prior permission was taken from the hospital authorities to conduct this study. Out of 400 cases, the records were scrutinized and completeness of the data available for 355 cases were included and analyzed for the present study. A total of 355 patients registered at the hospital were included in the study. The patients registered at this hospital were coming from all the parts of Al Qassim province which is a representative sample. Patients' case sheets were used as source of data. A detailed questionnaire including the information on socio economic conditions, personal history and clinical history was developed and these variables were analyzed in the study to give a clear picture of epidemiology of TB in the region. Findings were cross-checked with TB-Lab register, TB register, and TB cards of patients. Apart from demographic profile of the patients, the presenting symptoms, co-morbid conditions, diagnostic methods used and treatment regimen were also assessed. The cases were diagnosed by the medical officer of the hospital as per the WHO classification using the direct sputum examination and chest $\mathrm{X}$-ray. Montoux test was also done on all the patients.

\section{Ethical consideration and Statistical analysis:}

The study was approved by the Ethics and Research Committee of Qassim University and the hospital authorities of TB regional center. Data collected through structured questionnaires is entered and analyzed using Epi_info software (CDC Atlanta). Simple proportions and percentage were used and statistical analysis was done to see the association between TB and other variables.

\section{RESULTS}

A total of 355 patients with TB and other comorbid conditions accessing the TB services at regional TB center at Qassim comprised the study population. Of the 355 patients, Saudi ethnicity observed in $187(52.7 \%)$ patients compared to non Saudi $168(47.3 \%)$ as shown in figure 1. Out of the total non Saudi patients, the Indonesian patients contribute to $74(21 \%)$ followed by Indians $39(11 \%)$, Nepalese 12(3.4\%), Philippines \& Bangladesh with 9 cases each $(2.5 \%)$, and Pakistan 8(2.3\%). There 
were few patients from other countries like Sudan, Egypt, Jordan, Sri Lanka, Syria, Afghanistan and Morocco (including SAARC \& other countries) as shown in figure 2. Among the total number of patients diagnosed with TB, 150(42\%) were males and $205(58 \%$ ) were females (table 1) with the majority of participants $154(44 \%)$ being in the age group of $16-30$ years and $101(28.5 \%)$ being in the age group of $31-45$ years as shown in table No. 2 .

Nearly half of these cases 177(49.9\%) are pulmonary $\mathrm{TB}$, and the other half were divided between $170(47.9 \%)$ were extra-pulmonary cases and $8(2.2 \%)$ cases with both pulmonary and extrapulmonary involvement as shown in table 3 .

Patients included in this study with both pulmonary and extra pulmonary TB had various clinical presentations but they had complaints similar to the typical TB case presentation. The majority of patients $278(78.3 \%)$ were admitted with fever, $266(74.9 \%)$ had loss of appetite, 264(74.4\%) had loss of weight, $196(55.2 \%)$ complaining of cough with expectoration and $128(36.2 \%)$ were presenting with chest pain as shown in figure 3. In addition there were co-morbid conditions associated like Diabetes mellitus in 36 cases (10\%), and 12(3.5\%) patients were suffering from lung disease and $6(1.7 \%)$ suffering from chronic renal failure.

All the cases were treated with Directly Observed Treatment Short course (DOTS) regimen prescribed by World Health Organization. Out of total 355 patients on DOTS, $168(47.3 \%)$ patients were put under CAT I, 10 (2.8\%) CAT II and 175 cases (49.3\%) CAT III. There were 341(96\%) new

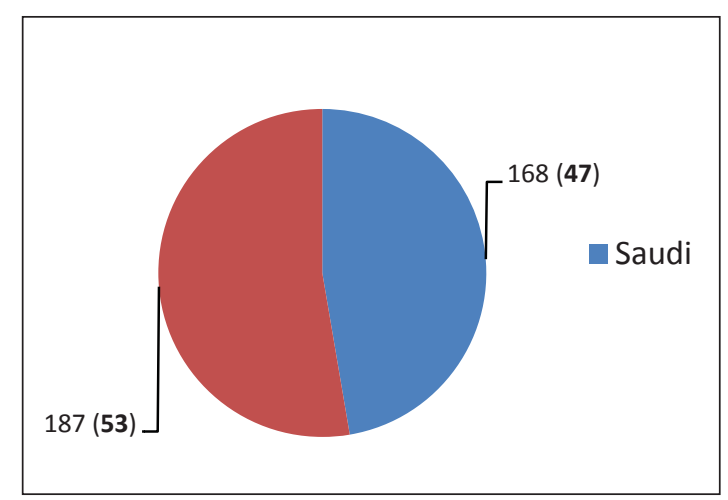

Figure 1. Ethnicity status of participants (percentages within brackets)

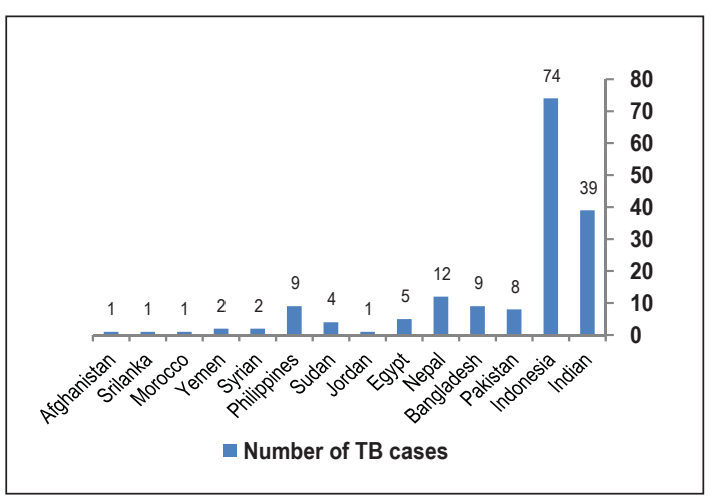

Figure 2. Ethnicity status of non Saudi participants

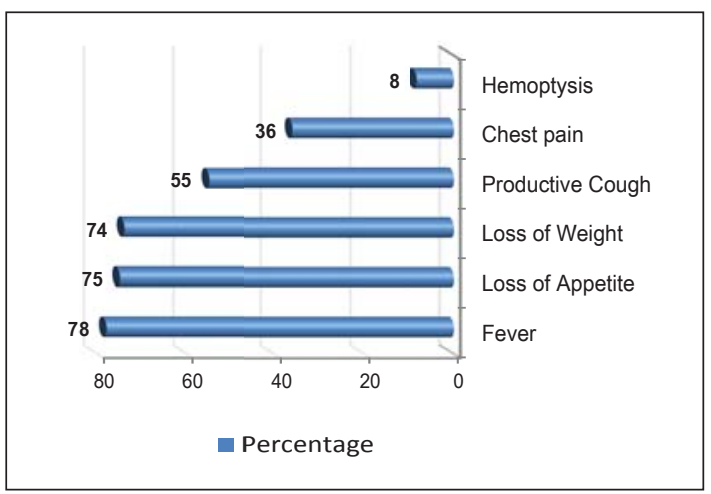

Figure 3. Distribution of common presenting complaints of TB cases

cases and $12(3.4 \%)$ relapse cases and $2(0.6 \%)$ defaulters among the total patients as shown in table 4.

Table 1. Distribution of study sample according to Sex

\begin{tabular}{|c|c|c|}
\hline Sex & Number & Percentage \\
\hline Male & 150 & $42 \%$ \\
\hline Female & 205 & $58 \%$ \\
\hline Total & 355 & $100 \%$ \\
\hline
\end{tabular}

Table 2. Distribution of study sample according to Age

\begin{tabular}{|c|c|c|}
\hline Age (yrs) & Number & Percentage \\
\hline $0-5$ & 8 & $2.3 \%$ \\
\hline $16-30$ & 154 & $43.3 \%$ \\
\hline $31-45$ & 101 & $28.5 \%$ \\
\hline $46-60$ & 36 & $10.1 \%$ \\
\hline$>60$ & 56 & $15.8 \%$ \\
\hline Total & 355 & $100 \%$ \\
\hline
\end{tabular}




\begin{tabular}{|c|c|c|}
\hline Type of Infection & Number & Percentage \\
\hline Pulmonary & 177 & $50 \%$ \\
\hline Extra-Pulmonary & 170 & $48 \%$ \\
\hline $\begin{array}{l}\text { Both pulmonary \& } \\
\text { extra pulmonary }\end{array}$ & 8 & $2 \%$ \\
\hline Total & 355 & $100 \%$ \\
\hline
\end{tabular}

Table 4. DOTS treatment status of the TB patients included in the study

\begin{tabular}{|l|l|l|}
\hline DOTS Regimen & Number & Percentage \\
\hline CAT I & 168 & $47.4 \%$ \\
\hline CAT II & 10 & $2.8 \%$ \\
\hline CAT III & 175 & $49.3 \%$ \\
\hline Other & 2 & $0.6 \%$ \\
\hline
\end{tabular}

\section{DISCUSSION}

Over the last few decades, considerable effort has been expended in industrialized countries to control the spread of TB. A lot of these processes were effective initially. ${ }^{12}$ In the USA, for instance, the incidence of TB decreased by $6 \%$ yearly. ${ }^{13}$ However, TB remains to be a major health concern throughout the world. It is critical for TB control and surveillance programs to address the burden of TB in certain population. Previous reports have indicated that the incidence of smear-positive TB in Saudi Arabia was estimated to be 20 per 100,000 populations ${ }^{14}$ Neighboring countries such as United Arab Emirates have similar rate of TB incidence.

Our study provides population-based data on the TB cases in Qassim region, Saudi Arabia from 2005 to 2009 . As shown approximately, $47.3 \%$ of TB cases included in this study are attributed for non Saudi patients indicating the important role that migrants play in TB epidemiology in Saudi Arabia. ${ }^{15}$ Majority of migrants were from TB-high burden countries, which is one of the important factors that contribute to resurgence of the disease. Therefore, screening migrants form countries endemic for TB is valuable to significantly reduce the spread of infection according the national guidelines. ${ }^{16}$ The high prevalence of extra-pulmonary TB in the study in comparison to overall of Saudi Arabia of $29.4 \%$ is probably due to the high number of females immigrating to Saudi Arabia as housemaids from the TB-high burden countries.

Our data suggested that approximately $73 \%$ of subjects participated in this study belong to the age group of $16-45$ years old proving that TB is a disease of economically productive age group among the low socio-economic strata of the society which goes in agreement with previously published study. ${ }^{17}$ Studies from South India have also shown that the prevalence of TB was higher in males than females at all ages: it was low in children under 10 years of age, increasing appreciably with age and reaching a peak between 20 and 40 years of age..$^{18}$

TB symptoms and clinical presentation of the present study were typical of TB cases with the majority of patients complained of Fever, loss of appetite, loss of weight, and cough with expectoration. Though other studies conducted in Africa \& Asia have documented the main presenting complaints were cough, weakness, loss of weight \& loss of appetite. In this current study, most of the patients belong to the low socio economic status, who are migrants either working as daily laborers or as house maids. One study from Malawi showed that higher socio-economic status was associated with $T B$, probably reflecting increased awareness and hence greater likelihood of diagnosis. ${ }^{19}$ Studies from China have revealed that TB was negatively correlated with per capita income; good household economic conditions were a protective factor. ${ }^{20-21}$

In our study, we found a strong association for TB in patients with chronic disease - there were co-morbid conditions associated like Diabetes mellitus in 36 cases (10\%), and 12(3.5\%) patients were suffering from lung disease and $6(1.7 \%)$ suffering from chronic renal failure. Few studies conducted in India and other places have demonstrated the association of TB with chronic disease like Diabetes, Hypertension \& coronary heart disease. ${ }^{22-24}$ The positive aspect of this study was that doctors working at the Regional Hospital were following the WHO-DOTS regimen for the diagnosis and treatment of even complicated cases of TB.

In conclusion, in the current study, we examined the incidence and the pattern of TB cases registered 
in Qassim TB center and the findings of this study revealed the high prevalence of TB among migrants mainly those from Southeast Asia. One of the important handicaps in TB control is late diagnosis. Pulmonary TB patients are often diagnosed many weeks after the onset of symptoms. This diagnostic delay allows for transmission of the disease to many contacts. Emphasis on timely contact tracing and treatment of those with active or latent disease is one of the most important aspects of TB control. Clinicians caring for TB patients complain of defects in this particular area. The National TB program needs to review its strategies, correct defects and improve TB control practices to make TB elimination an achievable goal. Further studies and continued surveillance of the TB infections are required to formulate plans for the effective management of TB.

\section{ACKNOWLEDGMENT}

Authors would like to thank the members of Tuberculosis Regional Center at Buraidah Central Hospital who gave their full cooperation and support for this study. Authors acknowledge the great help received from the scholars whose articles cited and included in references of this manuscript. The authors are also grateful to authors / editors / publishers of all those articles, journals and books from where the literature for this article has been reviewed and discussed. Authors are grateful to SAARC editorial board members and their team of reviewers who have helped to bring quality to this manuscript.

\section{REFERENCES}

1. The seventeenth global report on tuberculosis, Global Tuberculosis report 2012 http: / www.who. int / tb/ publications / global_report/gtbr12_annex4. pdf

2. Murray, C. and Feachem, R. Adult mortality in the developing world. Trans R Soc Trop Med Hyg 1990;84:21-22.

3. Styblo, K. Recent advances in epidemiological research in tuberculosis. Adv Tuberc Res 1980;20:1-63.
4. Murray C., DeJonghe E., Chum H., Nyangulu D., Salomao, A. and Styblo, K. Cost effectiveness of chemotherapy for pulmonary tuberculosis in three sub-Saharan African countries. Lancet 1991;338:1305-08.

5. Chamie G., Luetkemeyer A., Charlebois E. and Havlir, D. Tuberculosis as Part of the Natural History of HIV Infection in Developing Countries. Clin Infect Dis. 2010;15:50 Suppl 3:S245-54.

6. Harries, A. and Dye, C. Tuberculosis. Ann. Trop. Med. Parasitol 2006;415-31.

7. Dye, C. Global epidemiology of tuberculosis. Lancet 2006;367:934-40.

8. Bentrup, K. and Russell, D. Mycobacterial persistence: adaptation to a changing environment. Trends in Microbiol. 2001;597-605.

9. Khaled NA, Enarson D, Bousquet J. Chronic respiratory diseases in developing countries: The burden and strategies for prevention and management. Bulletin of the World Health Organization. Print ISSN 0042-9686. Geneva; 2001.

10. Health Statistics Book Year $2006 \mathrm{Ch}-1$ review of health situation, III: Chest Diseases.

11. Tuberculosis incidence trends in Saudi Arabia over 20 years: 1991-2010; Ibrahim Al-Orainey, Mogbil A Alhedaithy et al Annals of Thoracic Medicine 2013;8:3:148-52

12. Raviglione MC, Snider Jr. DE, Kochi A. Global epidemiology of tuberculosis. Morbidity and mortality of a worldwide epidemic JAMA 1995;273:220-6.

13. Snider Jr. DE, Roper WL. The new tuberculosis. N Engl J Med 1992;326:703-5.

14. Global tuberculosis control. WHO reports 19962002. Available at: www.emro.who.int/stb/ TBSituation-CountryProfilesaa.htm. Accessed Aug 1, 2009

15. Zaman R. Tuberculosis in Saudi Arabia: epidemiology and incidence of mycobacterium tuberculosis and other mycobacterial species. Tubercle 1991;72:43-9. 
16. Tulsky JP, White MC, Dawson C, Hoynes TM, Goldenson J, Schecter G. Screening for tuberculosis in jail and clinic follow-up for after release. Am J Public Health 1998;88: 223-6.

17. Bahl R, Singh B, Singh R. Prevalence of HIV infection among patients of pulmonary tuberculosis attending chest disease hospital, Jammu and Kashmir; Ind J of Community Med 2007;32:288289.

18. Balasubramanian R, Garg R, Santha $T$, et al. Gender disparities in tuberculosis: report from a rural DOTS programme in south India. Int J Tuberc Lung Dis 2004;8:323-32.

19. Glynn JR, Warndorff DK, Malema SS, et al. Tuberculosis: associations with HIV and socioeconomic status in rural Malawi. Trans R Soc Trop Med Hyg 2000;94:500-03.

20. 17 Liu JJ, Yao HY, Liu EY. Relationship between tuberculosis prevalence and socio-economic factors in China. Chinese. Zhonghua Liu Xing Bing Xue Za Zhi 2004;25:1032-34.
21. Liu JJ, Yao HY, Liu EY. Analysis of factors affecting the epidemiology of tuberculosis in China. Int $\mathrm{J}$ Tuberc Lung Dis 2005;9:450-54

22. Perez-Guzman C, Vargas M H, Torres-Cruz A, Perez-Padilla J R, Furuya M E, Villarreal-Velarde $\mathrm{H}$. Diabetes modifies the male:female ratio in pulmonary tuberculosis. Int $\mathrm{J}$ Tuberc Lung Dis 2003;7:354-58.

23. Ezung T, Devi NT, Singh NT, Singh TB. Pulmonary tuberculosis and diabetes mellitus-a study. J Indian Med Assoc 2002;100:376:378-79

24. N. Shetty, M. Shemko, M. Vaz, G. D'Souza. An epidemiological evaluation of risk factors for tuberculosis in South India: a matched case control study. Int J Tuberc Lung Dis 2006;10:80-86 